\title{
Kontingente Bauchgefühle
}

\author{
Urteilen und Entscheiden in der Sozialraumarbeit im Kontext von Programmierungen und \\ ökonomisierter Wissensproduktion
}

\begin{abstract}
Professionelles Arbeiten mit Bezug zu "Sozialraum” ist äußert vielgestaltig. Der Beitrag skizziert einige Überlegungen, wie sich dennoch Rückschlüsse auf Prozesse und Kontexte des Entscheidens und Urteilens bei Fachkräften in sozialräumlichen Arbeitsfeldern ziehen lassen. Ausgehend von Befunden der Urteilsund Entscheidungsforschung wird hierbei zunächst die Bedeutung von Subjektivierungsprozessen und im Anschluss daran, die Rolle von Programmen und der Einfluss der Beratungswirtschaft beleuchtet. Der Beitrag schließt mit Anregungen zu weiterführenden Forschungsperspektiven.
\end{abstract}

$\mathrm{D}$ ie Beschäftigung mit Prozessen der professionellen Entscheidungsfindung und Urteilsbildung in der Sozialen Arbeit ist bedeutsam, da aus diesen mitunter sehr weitreichende Folgen für Adressat_innen bzw. Nutzer_innen resultieren können. In der Gemeinwesenarbeit und im weiteren Sinne, der Sozialraumarbeit, können solche Prozesse erhebliche Auswirkungen auf die Gestaltung des örtlichen Zusammenlebens und auf die strukturellen Bedingungen der Lebensführung von Einzelpersonen, Familien oder lokalen Gemeinschaften haben.

Aufgrund des komplexen Gefüges von (sozial-)rechtlichen Aufträgen, fachlichen Standards, normativen oder politischen Positionierungen, Beschäftigungsformen und Praxiskonstellationen, ist es jedoch kaum möglich, verallgemeinernde Aussagen zu Entscheidungsfindungsund Urteilsbildungsprozessen der Professionellen zu treffen. Allein das Feld der Sozialraumarbeit erweist sich im Hinblick auf Arbeitsschwerpunkte, Methoden,
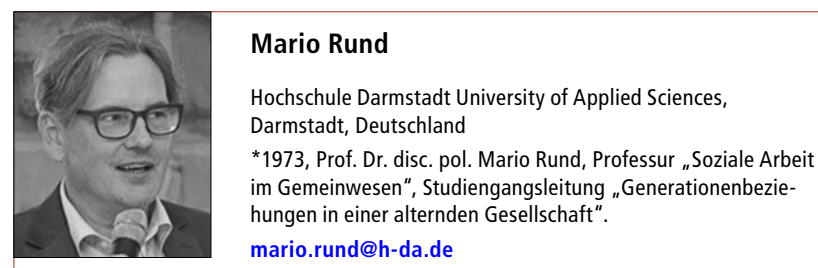

Zusammenfassung Praktiken des Entscheidens und Urteilens sind mit Subjektverhältnissen verknüpft. Für die Forschung sind daher für Subjektivierungsprozesse relevante Kontexte und Einflüsse zu fokussieren. Hinsichtlich der Sozialraumarbeit gehören hierzu bestimmte Anrufungen und Rationalitäten, die über verschiedene Programme mit Bezug zu Gemeinwesen, Sozialraum und Raum transportiert werden.

Schlüsselwörter Urteilsbildung, Subjektivierung, Progammanalyse, Beratungswirtschaft, Sozialraumorientierung, Gemeinwesenarbeit/Sozialraumarbeit
Qualifikation und disziplinäre Herkunft des Personals (Soziale Arbeit, Soziologie, Stadt- und Raumplanung u. a.), der organisatorischen Einbettung (Kommunen, sog. Freie Träger, Wohnungsunternehmen, Planungsbüros, Stadtteilvereine u. a.) als ungemein vielfältig. Trotz dieser Vielfalt könnte die Erforschung von Entscheidungsfindungs- und Urteilsbildungsprozessen dazu beitragen, Fachkräfte für Kontexte und Einflüsse ihrer Entscheidungen und Urteile zu sensibilisieren, die ihnen üblicherweise nicht reflexiv verfügbar sind. Allerdings wäre auch zu prüfen, inwieweit solche Untersuchungen die professionelle Entscheidungsfindung und Urteilsbildung in der Sozialraumarbeit selbst beeinflussen und die Forschungsergebnisse, Begehrlichkeiten nach einer wirkungsbezogenen Neuvermessung des Arbeitsfeldes befördern könnten.

\section{Professionelle Subjektivierungen}

Ein zentraler Befund der Urteils- und Entscheidungsforschung besteht darin, dass professionelles Urteilen und Entscheiden nicht der Logik reiner, durch KostenNutzen-Kalküle geprägter, rationaler Handlungen entsprechen (Bastian und Schrödter 2014, S. 291). Urteile sind vielmehr soziale Konstruktionen als Antwort auf die kontingenten und paradoxalen Anforderungen professionell zu bearbeitender Probleme (ebd. S. 293). Diese Konstruktionen basieren auf bestimmten Wissensformen sowie deren Reflexion und werden durch Intuition und Haltungen beeinflusst (Bastian 2019, S. 87).

Diese Wissens- und Reflexionsformen sowie Haltungen sind mit spezifischen Subjektverhältnissen der Fachkräfte verbunden, die sich etwa in professionellen Selbstverständnissen widerspiegeln. Im Hinblick auf die unterschiedlichen Weisen, wie Fachkräfte ihre Adressat_ innen und die Beziehung zwischen sich und ihnen interpretieren, kann beispielsweise zwischen einem responsivem, klinischem, manageriellem und solidarischem 
Selbstverständnis von Professionalität unterschieden werden (Kunstreich 1997/2014, S. 355 ff.). Über organisationale oder fachliche Faktoren hinaus stellen die Deutungen der eigenen professionellen Rolle, die politische Positionierung, die Konstruktion von Adressat_innen oder die Wahrnehmung von Handlungs- und Entscheidungsspielräumen, wesentliche Aspekte professioneller Subjektivität dar. Um Rückschlüsse über Relevanzsetzungen, Kategorisierungen, Klientifizierungen, Entscheidungsheuristiken und -praktiken zu erhalten, ist es daher sinnvoll, Subjektivierungsprozesse von Fachkräften und ihren Umgang mit an sie adressierte „Subjektivierungsangebote“ in den Blick zu nehmen.

Im Prozess der Selbstkonstitution spielen Deutungsofferten, wie sie über diskursiv hergestellte und reproduzierte Wissensordnungen und Wahrheitsregime formuliert werden, eine zentrale Rolle. Die paradoxe Gleichzeitigkeit von gesellschaftlicher Produktion und Selbstformierung des Subjektes hat Althusser (1977, S. 140 ff.) im Rahmen seiner Ideologietheorie mit dem Konzept der „Anrufung“ („Interpellation“) beschrieben. Demzufolge werden durch Ideologie „aus der Masse der Individuen Subjekte, rekrutiert' [...] oder diese Individuen in Subjekte transformiert" (Althusser 1977, S. 142).

\section{Programmierte Wirklichkeit}

Als materialisierte gesellschaftliche, politische und fachliche Diskurse vermitteln Programme bestimmte Wissensbestände und Wirklichkeitsbeschreibungen, in denen sich durch diese Programme Angerufene selbst erkennen, entlang derer sie ihre Subjektivität formen und bestimmte Perspektiven auf die soziale und materiale Umwelt einnehmen können. Sozialraumarbeit wird fast ausschließlich auf Basis von Fach- und Förderprogrammen auf Kommunal-, Landes-, Bundes- oder EUEbene realisiert (z. B. „Soziale Stadt“ bzw. „Sozialer Zusammenhalt“, „Wohnen in Nachbarschaften“, „Lokales Kapital für soziale Zwecke“, „Jugend stärken im Quartier"). Neben der akademischen Ausbildung sind solche Programme daher als bedeutsame Ressourcen der Konstitution von „Professionssubjekten“ und somit auch ihren Entscheidungsfindungs- und Urteilspraktiken anzusehen. Denn Programme verfügen im Allgemeinen über das Potential, soziale Praktiken, Techniken, Gegenstandsbereiche und Subjektivität der Individuen zu formen (Kessl und Krasmann 2019, S. 283; Bröckling et al. 2004, S. 12 f.), aber auch Formen der Entscheidungsfindung und Urteilsbildung hervorzubringen. Programmmaterialien können daher Realität gestalten, indem sie diese in bestimmter Weise beschreiben oder Probleme definieren und diese auf spezifische Art von anderen abgrenzen sowie Möglichkeiten zur deren Bewältigung vorschlagen. In diesem Sinne sind Programme deskriptiv und darin zugleich präskriptiv sowie performativ bzw. „realitätsmächtig“. Sie bieten Technologien und Deutungsschemata an, mit denen Individuen sich selbst führen (sollen) und mit deren Hilfe individualisierte Subjekte hervorgebracht werden bzw. sich selbst hervorbringen (Foucault 1982/2005, S. 275). Programme formulieren Anweisungen oder Empfehlungen, „wie Menschen zu behandeln sind und wie sie sich zu verhalten haben, um als Individuen gelten zu können “ (Bröckling 2007, S. 24 f.). Damit legen Programme nahe, wie sich Individuen als Subjekte innerhalb von Machtbeziehungen zu verstehen haben und wie sie denken und sprechen, aber auch urteilen, entscheiden und handeln sollen. Indem sie bestimmte Subjektpositionen und -modelle skizzieren, rufen Programme Individuen an, sich als Subjekte zu begreifen und auf spezifische Art, etwa „kreativ und klug, unternehmerisch und vorausschauend, sich selbst optimierend und verwirklichend usw." (Bröckling et al. 2004, S. 12) zu verhalten und entsprechende Entscheidungen zu treffen.

Im Rahmen von Subjektivierungsregimen eröffnen und strukturieren Programme Möglichkeitsräume, innerhalb derer dem Subjekt die Freiheit zum Handeln gegeben wird. Daher übersetzen sich Programme keineswegs bruchlos in individuelles Verhalten oder bringen vorbestimmte Subjektivitäten automatisch hervor (Bröckling 2007, S. 40). Programme formen vielmehr „die Realität innerhalb einer Rationalität, in den Weisen des Denkens, des Urteilens und der Einschätzung, die sich in Praktiken einschreiben “ (Krasmann 2003, S. 74). Dabei drücken Programme nicht nur Wünsche und Absichten aus. Sie setzen vielmehr implizite Wissensordnungen und somit bestimmte Denk- und Redeweisen voraus, entlang derer Realität auf spezifische Weise „programmierbar" wird (Lemke 1997, S. 147). Hierdurch können professionelle Entscheidungsfindungs- und Urteilsbildungsprozesse in einer Weise beeinflusst werden, die eine Ausrichtung der Sozialen Arbeit, und somit auch der Sozialraumarbeit, an Diskursen zur postwohlfahrtsstaatlichen oder „neo-sozialen Neubestimmung “ (Rose 2000) bzw. „Neuerfindung“ des Sozialen (Lessenich 2008) unterstützt.

\section{Marktgetriebene Wissensproduktion}

Eine zentrale Rolle bei der Reproduktion solcher Programmrationalitäten und entsprechender Subjektivierungsofferten spielt die sozial- und ingenieurwissenschaftliche Beratungswirtschaft, die im Zuge von Outsourcing ehemals staatlich verantworteter Aufgaben im Kontext der unternehmerisch umgebauten öf- 


\section{Extrablick: Urteilsbildung in der Sozialen Arbeit}

fentlichen Verwaltung (Neue Steuerung) eine bemerkenswerte Konjunktur erlebt. Private Unternehmen oder (An-)Institute öffentlicher Bildungseinrichtungen entwickeln Programme inklusive „Werkzeugkästen“, Handlungsempfehlungen und Maßnahmenkataloge zur Bewältigung von Problemen, die sie als solche häufig selbst erst beschrieben haben. Vor dem Hintergrund eines problematischen Steuerungsoptimismus (Merchel 2018, S. 9) basiert das Geschäftsmodell der akademischen Beratungsunternehmer_innen auf der Suggestion eines Gesellschaftsmodells, in dem „scheinbar ein gezielter Knopfdruck [genügt], um unterschiedliche Lebenswirklichkeiten, Bedürfnisse und Mächtigkeiten zur stillen Koexistenz zu neutralisieren" (Fritsche und Reutlinger 2015, S. 202). Denn die Wettbewerbslogik des „Berater-Kapitalismus“ (Resch 2005) erfordert, fachliche Wissensbestände zu vulgarisieren und zu kommodifizieren, d. h. in leicht zu vermarktende und vermeintlich genau bewertbare bzw. messbare Leistungen und Produkte zu verwandeln. Mitunter bis an die Grenze der Unredlichkeit müssen Forschung und akademische Wissensproduktion unternehmerischen Kalkülen unterworfen werden um sich im kompetitiven Beratungsmarkt zu positionieren und Folgeaufträge zu generieren. Statt als kritische Begleitung, fungiert Wissenschaft hierbei oft eher als Legitimationsinstrument selbst für fragwürdigste politische Projekte und administrative Routinen. Schließlich wäre verantwortungsvolle, unabhängige oder gar kritische Wissenschaft, die etwa die unablässige Reproduktion sozialer und räumlicher Ungleichheit und Exklusion infolge unternehmerischer Stadtpolitik und neoliberaler Sozialpolitik problematisiert, ein existenzbedrohender Wettbewerbsnachteil im Beratungsmarkt. Wie sich am Beispiel von Programmen zur „Sozialraumorientierung “ zeigt, können die beratungswirtschaftlichen Produktionslogiken erhebliche Folgen für die Weisen der professionellen Entscheidungsfindung und Urteilsbildung in der Sozialraumarbeit haben.

\section{Sozialräumliche Programmierungen}

Dominierende Sozialraumprogramme, etwa mit Fokus auf Kinder- und Jugend, Integration oder Quartiersentwicklung, richten sich an der Steuerungslogik von Kommunen oder Ministerien aus und verändern zugleich die Fachlichkeit (Düring und Peters 2016, S. 1073; Kessl und Reutlinger 2018, S. 1085) und somit auch die Entscheidungs- und Urteilspraktiken in der Sozialraumarbeit. Denn neben theoretisch verkürzten, territorialen Sozialraumkonzeptionen (Landhäußer 2009, S. 132) wird in den Programmen zumeist mit Problembeschreibungen operiert, die stigmatisierende Diagnosen von „Rückständigkeit“ und „Entwicklungsdefiziten“ von Quartieren und Bewohner_innen aufruhen (May 2016a, S. 43 f.). Die Lösungsvorschläge, die einflussreiche Rahmensetzungen für professionelle Entscheidungen und Urteile darstellen, beziehen sich dabei häufig auf Konzepte normalisierender Prävention, responsibilisierender Aktivierung, individualisierendem und psychologisierendem Empowerment oder machtnaiver Governance. Insbesondere im Kontext einer solidarischen Professionalität, die sich an den widersprüchlichen Äußerungen und Handlungserwartungen der Adressat_innen ausrichtet (Kunstreich 1997/2014, S. 367 f.), können die über Programme gesteuerten Ressourcen zwar weiterhin „transversal“, d. h. quer zu den Kommunikations- und Kooperationsgeflechten herrschender Institutionen für die Arbeit am Gemeinwesen (May 2016b) eingesetzt werden. Dennoch verwundert es kaum, wenn sich Fachkräfte angesichts dominierender programmatischer Problembeschreibungen und Lösungsvorschläge etwa als „Kümmerer“ bezeichnen, in absoluten, territorialen Raumbegriffen denken, sozialstrukturell höchst selektive Partizipationsprozesse begleiten und ihr Praxishandeln durch Beschränkung auf ein Planungsraum oder Quartier bzw. von fachlichen Sozialraumautismus geprägt ist. Für eine kritisch-reflexive Sozialraumarbeit charakteristische professionelle Entscheidungen, etwa für die angemessene Würdigung von Wissensbeständen und die Begleitung von Aktivitäten lokaler Akteur_innen (Fritsche und Reutlinger 2015, S. 203), der Unterstützung „alternativer Quartiersphilosophien“ (May 2016a, S. 47) oder die Förderung „radikaler Demokratie“ (Alinsky 1971/1989) werden somit eher unwahrscheinlich.

\section{Fazit und Forschungsperspektiven}

Im beratungswirtschaftlich-administrativ-politischen Komplex werden über Programme wesentliche formale Rahmenbedingungen für die Sozialraumarbeit gesetzt, aber auch inhaltliche Deutungs- und Bewertungskriterien formuliert - und somit Selbstkonstituierung, Selbstpositionierung und Urteilspraktiken der Fachkräfte in diesem Arbeitsfeld präformiert. Problematisierungen der Rolle von (sozial-)politischen und fachlichen Programmen sowie der Programmproduktion durch die Beratungswirtschaft, bieten Fachkräften der Sozialraumarbeit eine Sensibilisierung für wesentliche Faktoren der (Selbst-)Formierung als „Professionssubjekt“ und somit für die Reflexion ihrer Entscheidungs- und Urteilspraktiken.

$\mathrm{Zu}$ diesen Problematisierungen könnten diskurstheoretisch informierte Programmanalysen beitragen (Kessl und Krasmann 2019, S. 281 ff.). Diese vermögen zwar nicht die konkrete Realisierung von Programmen zu 
prognostizieren. Aber sie können beschreiben, wie Individuen adressiert werden, welches Verhalten ihnen zugemutet, zugetraut oder von ihnen erwartet wird und in welcher Weise andere oder sie selbst auf ihr Verhalten in geregelter Weise Einfluss nehmen (sollen) (Bröckling et al. 2004, S. 12). Daher könnten auch Adressierungsanalysen im Rahmen der Subjektivierungsforschung (Geimer et al. 2019) hierbei wertvolle Einsichten liefern. Denn etwa mit Althussers Interpellationskonzept, Foucaults Diskurs- und Machtbegriff sowie Butlers Theorie zur Performativität können (programmatische) Anrufungen in Dimensionen wie Macht, Wissen und Selbstverhältnis untersucht werden.

Eingegangen. 7. April 2021

\section{Angenommen. 11. Mai 2021}

Funding. Open Access funding enabled and organized by Projekt DEAL.

Open Access. Dieser Artikel wird unter der Creative Commons Namensnennung 4.0 International Lizenz veröffentlicht, welche die Nutzung, Vervielfältigung, Bearbeitung, Verbreitung und Wiedergabe in jeglichem Medium und Format erlaubt, sofern Sie den/die ursprünglichen Autor(en) und die Quelle ordnungsgemäß nennen, einen Link zur Creative Commons Lizenz beifügen und angeben, $\mathrm{ob}$ Änderungen vorgenommen wurden.

Die in diesem Artikel enthaltenen Bilder und sonstiges Drittmaterial unterliegen ebenfalls der genannten Creative Commons Lizenz, sofern sich aus der Abbildungslegende nichts anderes ergibt. Sofern das betreffende Material nicht unter der genannten Creative Commons Lizenz steht und die betreffende Handlung nicht nach gesetzlichen Vorschriften erlaubt ist, ist für die oben aufgeführten Weiterverwendungen des Materials die Einwilligung des jeweiligen Rechteinhabers einzuholen.

Weitere Details zur Lizenz entnehmen Sie bitte der Lizenzinformation auf http://creativecommons.org/licenses/by/4.0/deed.de.

\section{Literatur}

Alinsky, S. (1989). Rules for radicals. New York: Vintage Books.

Althusser, L. (1977). Ideologie und ideologische Staatsapparate. In L. Althusser (Hrsg.), Ideologie und ideologische Staatsapparate (S. 108-153). Hamburg, Berlin: VSA.

Bastian, P. (2019). Sozialpädagogische Entscheidungen. Stuttgart: UTB.

Bastian, P., \& Schrödter, M. (2014). Professionelle Urteilsbildung in der Sozialen Arbeit. Soziale Passagen, 2, 275-297.

Bröckling, U. (2007). Das unternehmerische Selbst. Frankfurt am Main: Suhrkamp.

Bröckling, U., Krasmann, S., \& Lemke, T. (2004). Einleitung. In U. Bröckling, S. Krasmann \& T. Lemke (Hrsg.), Ein Glossar der Gegenwart (S. 9-14). Frankfurt am Main: Suhrkamp.

Düring, D., \& Peters, F. (2016). Sozialraumorientierung. In W. Schröer, N. Struck \& M. Wolff (Hrsg.), Handbuch Kinder- und Jugendhilfe (S. 10671090). Weinheim, Basel: Juventa.

Foucault, M. (2005). Subjekt und Macht. In M. Foucault (Hrsg.), Schriften in vier Bänden. Bd. IV: 1980-1988 (S. 269-294). Frankfurt am Main: Suhrkamp.
Fritsche, C., \& Reutlinger, C. (2015). Der öffentliche Raum ist (k)ein Problem. In R. Kemper \& C. Reutlinger (Hrsg.), Umkämpfter öffentlicher Raum (S. 193-205). Wiesbaden: Springer VS.

Geimer, A., Amling, S., \& Bosančić, S. (2019). Subjekt und Subjektivierung. Wiesbaden: Springer VS.

Kessl, F., \& Krasmann, S. (2019). Sozialpolitische Programmierungen. In F. Kessl \& C. Reutlinger (Hrsg.), Handbuch Sozialraum, Sozialraumforschung und Sozialraumarbeit (S. 277-297). Wiesbaden: Springer VS.

Kessl, F., \& Reutlinger, C. (2018). Sozialraumorientierung. In K. Böllert (Hrsg.), Kompendium Kinder- und Jugendhilfe (S. 1067-1092). Wiesbaden: Springer VS.

Krasmann, S. (2003). Die Kriminalität der Gesellschaft. Konstanz: UVK.

Kunstreich, T. (2014). Grundkurs Soziale Arbeit. Hamburg: Agentur des Rauhen Hauses. 2. Bd

Landhäußer, S. (2009). Communityorientierung in der Sozialen Arbeit. Wiesbaden: VS.

Lemke, T. (1997). Eine Kritik der politischen Vernunft. Hamburg: Argument.

Lessenich, S. (2008). Die Neuerfindung des Sozialen. Bielefeld: transcript.

May, M. (2016a). Soziale Arbeit in der unternehmerischen Stadt. In P. Oehler, N. Thomas \& M. Drilling (Hrsg.), Soziale Arbeit in der unternehmerischen Stadt (S. 41-50). Wiesbaden: Springer VS.

May, M. (2016b). Soziale Arbeit als Arbeit am Gemeinwesen. Opladen, Berlin, Toronto: Barbara Budrich.

Merchel, J. (2018). Steuerung in der Sozialen Arbeit. Sozialmagazin, 9-10, 6-13.

Resch, C. (2005). Berater-Kapitalismus oder Wissensgesellschaft? Münster: Westfälisches Dampfboot.

Rose, N. (2000). Tod des Sozialen? In U. Bröckling, S. Krasmann \& T. Lemke (Hrsg.), Gouvernementalität der Gegenwart (S. 72-109). Frankfurt am Main: Suhrkamp. 